\title{
Certain Aspects of Univalent Function with Negative Coefficients Defined by Bessel Function
}

\author{
Chellakutti Ramachandran $^{1}{ }^{*}$, Kaliyaperumal Dhanalakshmi ${ }^{1}$ and Lakshminarayanan \\ Vanitha ${ }^{1}$. \\ ${ }^{I}$ Department of Mathematics; University College of Engineering; Villupuram; Anna University; Tamilnadu; India.
}

\begin{abstract}
Key words: In recent years, applications of Bessel functions have been effectively used in the modelling of chemical engineering processes and theory of univalent functions.In this paper, we study a new class of analytic and univalent functions with negative coefficients in the open unit disk defined by Modified Hadamard product with Bessel function. We obtain coefficient bounds and exterior points for this new class.
\end{abstract}

2010 AMS Mathematics Subject Classification: Primary 30C45, Secondary 30C50, 30C80.

Keywords:Analytic function, Univalent function, Extreme point, Bessel function, Subordination and Hadamard Product.

\footnotetext{
*Author for correspondence: crjsp2004@yahoo.com
} 


\section{INTRODUCTION}

Many important functions in applied sciences are defined via improper integrals or series (or infinite products). The general name of these important functions are called special functions. Bessel functions are important special functions which are playing the important role in studying solutions of differential equations. Especially, the linear PDE describing various chemical transfer processes, allow the exact solution expressed in terms of one special kind of Bessel's functions and they are associated with a wide range of problems in important areas of mathematical physics, modelling of transfer processes in chemical engineering as well as in the related fields like hydrodynamics, heat transfer, diffusion, bioprocesses and so on. By using the method of seperation of variables, exact solution in terms of Bessel function can be used to calculate several important parameters which are needed in design and construction of chemical engineering apparatuses and equipment like heat exchangers and their components. Typical example for the efficiency calculation is applied in Brazilian powdered milk plant [11]. In another case when the Bessel functions arises is heat transfer modelling which considered in [6]. Here the problem of cross-flow streaming of heated object with large value of length to diameter ratio (like thermoanemometer) is solved for small Pe numbers using the theory of analytic functions.

Recently Deniz [9] has studied the following: The generalized Bessel function of the first kind of order $u$ , is defined as function $\omega_{u, b, c}(z)$, has the familiar representation as follows

$$
\omega_{u, b, c}(z)=\sum_{n=0}^{\infty} \frac{(-c)^{n}}{n ! \Gamma\left(u+n+\frac{b+1}{2}\right)}\left(\frac{z}{2}\right)^{2 n+u}, z \in \mathrm{C}
$$

Where $\Gamma$ stands for the Euler gamma function. The series (1.1) permits the study of Bessel function in a unified manner. It is called the particular solution of the following second-order linear homogeneous differential equation (see for details[5]):

$$
z^{2} \omega^{\prime \prime}(z)+b z \omega^{\prime}(z)+\left[c z^{2}-u^{2}+(1-b) u\right] \omega(z)=0, u, b, c \in \mathrm{C} .
$$

. Also in Deniz et al. [7] and Deniz [8] (see also[1, 3, 4, 10] studied the function $\varphi_{u, b, c}(z)$ defined, in terms of the generalized Bessel function $\omega_{u, b, c}(z)$ by the transformation

$$
\varphi_{u, b, c}(z)=2^{u} \Gamma\left(u+\frac{b+1}{2}\right) z^{1-\frac{u}{2}} \omega_{u, b, c}(\sqrt{z}) .
$$

By using the well known Pochhammer symbol (or the shifted factorial) $(\lambda)_{\mu}$ is defined in terms of the Euler $\Gamma$ function, by

$$
(\lambda)_{\mu}=\frac{\Gamma(\lambda+\mu)}{\Gamma(\lambda)}=\left\{\begin{array}{cc}
1, & \mu=0 \\
\lambda(\lambda+1) \ldots(\lambda+n-1), & \mu \in \mathrm{N}
\end{array}\right.
$$

it being understood conventionally that $(\lambda)_{0}=1$. We obtain the following series representation for the function $\varphi_{u, b, c}(z)$ given by (1.3):

$$
\varphi_{u, b, c}(z)=z+\sum_{n=1}^{\infty} \frac{(-c)^{n}}{4^{n}(\kappa)_{n} n !} z^{n+1}, z \in \mathrm{C}
$$

where $\kappa=u+\frac{b+1}{2} \notin \mathrm{Z}_{0}^{-}, \quad \mathrm{N}=\{1,2, \ldots\}$ and $\mathrm{Z}_{0}^{-}=\{0,-1,-2, \ldots\}$. For convenience, we write $\varphi_{\kappa, c}(z)=\varphi_{u, b, c}(z)$. Next, we introduce a operator $B_{\kappa}^{c}: \mathrm{S} \rightarrow \mathrm{S}$, which is defined by the Hadamard product 


$$
\begin{aligned}
B_{\kappa}^{c} f(z) & =\varphi_{\kappa, c}(z) * f(z)=z+\sum_{n=1}^{\infty} \frac{(-c)^{n} a_{n+1}}{4^{n}(\kappa)_{n} n !} z^{n+1} \\
& =z+\sum_{n=2}^{\infty} \frac{(-c)^{n-1} a_{n}}{4^{n-1}(\kappa)_{n-1}(n-1) !} z^{n}=z+\sum_{n=2}^{\infty} D(c, \kappa, n) a_{n} z^{n}
\end{aligned}
$$

where $D(c, \kappa, n)=\frac{(-c)^{n-1}}{4^{n-1}(\kappa)_{n-1}(n-1) !}$

It is easy to verify that from $(1.5) z\left[B_{\kappa+1}^{c} f(z)\right]^{\prime}=\kappa B_{\kappa}^{c} f(z)-(\kappa-1) B_{\kappa+1}^{c} f(z)$

where $\kappa=p+\frac{b+1}{2} \notin \mathrm{Z}_{0}^{-}$. In fact the function $B_{\kappa}^{c}$ given by (1.5) is an elementary transformation of the generalized hypergeometric function. Hence, it is easy to see that $B_{\kappa}^{c} f(z)=z_{0} F_{1}\left(\kappa ; \frac{-c}{4} z\right)^{*} f(z)$ and also $\varphi_{\kappa, c}\left(\frac{-c}{4} z\right)=z_{0} F_{1}(\kappa ; z)$. Let $\mathrm{A}$ be the class of all analytic functions

$$
f(z)=z+\sum_{n=2}^{\infty} a_{n} z^{n}
$$

in the unit disk $U=\{z:|z|<1\}$. Let $\mathrm{S}$ be the subclass of $\mathrm{A}$ consisting of univalent functions. Suppose for $0 \leq \eta<1, \mathrm{~S}^{*}(\eta)$ and $\mathrm{C}(\eta)$ denote the subclasses of $\mathrm{A}$ consisting of functions which satisfy the following inequalities: $\mathfrak{R}\left(\frac{z f^{\prime}(z)}{f(z)}\right)>\eta$, and $\mathfrak{R}\left(1+\frac{z f^{\prime \prime}(z)}{f^{\prime}(z)}\right)>\eta$,

are, respectively, starlike and convex of order $\eta$ in $\mathrm{U}$ In particular, we set $\mathrm{S}^{*}(0)=\mathrm{S}^{*}$ and $\mathrm{C}(0)=\mathrm{C}$. Let $\mathrm{T}$ denote the subclasses of $\mathrm{S}$ consisting of functions $f(z)$ given by

$$
f(z)=z-\sum_{n=2}^{\infty} a_{n} z^{n}, a_{n} \geq 0
$$

with negative coefficients. Silverman[13] introduced and investigated the following subclasses of the function class $\mathrm{T}: \mathrm{T}^{*}(\eta)=\mathrm{S}^{*}(\eta) \cap \mathrm{T}$ and $\mathrm{C}^{*}(\eta)=\mathrm{C}(\eta) \cap \mathrm{T}, 0 \leq \eta<1$

For $f \in \mathrm{A}$ given by (1.6) and $g \in \mathrm{A}$ is given by $g(z)=z+\sum_{n=1}^{\infty} b_{n+1} z^{n+1}$, the Hadamard product (or convolution) of $f(z)$ and $g(z)$ is given by $(f * g)(z)=z+\sum_{n=1}^{\infty} a_{n+1} b_{n+1} z^{n+1}=(g * f)(z), \quad z \in \mathrm{U}$ Recently Shanmugam et al.[12] and [15] have studied the following:

Definition 1.1 Let $c>1,0 \leq \lambda<1, k \geq 0,0 \leq \eta<1$ and $z \in \mathrm{U}$, a function $f \in \mathrm{T}$ is said to be in the class $\mathrm{UB}(\lambda, \eta, k, c)$ if it satisfies the following inequality:

$$
\mathfrak{R}\left[\frac{z F^{\prime \prime}(z)}{F^{\prime}(z)}\right]>k\left|\frac{z F^{\prime \prime}(z)}{F^{\prime}(z)}-1\right|+\eta
$$

where $F(z)=(1-\lambda)\left(B_{\kappa}^{c} f(z)\right)+\lambda z\left(B_{\kappa}^{c} f(z)\right)^{\prime}$.

Lemma 1.2 [2] Let $w=u+i v$. Then $\operatorname{Re}(w)>\sigma$ if and only if $|w-(1+\sigma)| \leq|w+(1-\sigma)|$ 
Lemma 1.3 [2] Let $w=u+i v$ and $\sigma, \gamma$ are real number. Then $\operatorname{Re}(w)>\sigma|w-1|+\gamma$ if and only if $\operatorname{Re}\left[w\left(1+\sigma e^{i \phi}\right)-\sigma e^{i \phi}\right]>\gamma$.

2 Coefficient Bounds and Extreme Points

We obtain the necessary and sufficient condition and extreme points for the function $f(z)$ in the class $\mathrm{UB}(\lambda, \eta, k, c)$.

Theorem 2.1 Let $c>1,0 \leq \lambda<1, k \geq 0,0 \leq \eta<1$ and $z \in \mathrm{U}$. The function $f(z)$ defined by equation (1.6) is in the class $\mathrm{UB}(\lambda, \eta, k, c)$ if and only if

$$
k+\sum_{n=2}^{\infty} n(n(1+k)-(\eta+2 k))(1-\lambda+n \lambda) D(c, \kappa, n) \leq 1-\eta
$$

Proof. From the definition, we have

$$
\begin{gathered}
\mathfrak{R}\left\{\frac{\left(B_{\kappa}^{c} f(z)\right)^{\prime}+(1+2 \lambda) z\left(B_{\kappa}^{c} f(z)\right)^{\prime \prime}+\lambda z^{2}\left(B_{\kappa}^{c} f(z)\right)^{\prime \prime \prime}}{\left(B_{\kappa}^{c} f(z)\right)^{\prime}+\lambda z\left(B_{\kappa}^{c} f(z)\right)^{\prime \prime}}-1\right\} \\
\geq k\left\{\frac{\left(B_{\kappa}^{c} f(z)\right)^{\prime}+(1+2 \lambda) z\left(B_{\kappa}^{c} f(z)\right)^{\prime \prime}+\lambda z^{2}\left(B_{\kappa}^{c} f(z)\right)^{\prime \prime \prime}}{\left(B_{\kappa}^{c} f(z)\right)^{\prime}+\lambda z\left(B_{\kappa}^{c} f(z)\right)^{\prime \prime}}-2\right\}+\eta
\end{gathered}
$$

From Lemma 1.3, we have

$$
\operatorname{Re}\left\{\frac{\left(B_{\kappa}^{c} f(z)\right)^{\prime}+(1+2 \lambda) z\left(B_{\kappa}^{c} f(z)\right)^{\prime \prime}+\lambda z^{2}\left(B_{\kappa}^{c} f(z)\right)^{\prime \prime \prime}}{\left(B_{\kappa}^{c} f(z)\right)^{\prime}+\lambda z\left(B_{\kappa}^{c} f(z)\right)^{\prime \prime}}\left(1+k e^{i \phi}\right)-2 k e^{i \phi}\right\} \geq \eta
$$

$-\pi \leq \phi \leq \pi$, or equivalently

$\mathfrak{R}\left\{\frac{\left[\left(B_{\kappa}^{c} f(z)\right)^{\prime}+(1+2 \lambda) z\left(B_{\kappa}^{c} f(z)\right)^{\prime \prime}+\lambda z^{2}\left(B_{\kappa}^{c} f(z)\right)^{\prime \prime \prime}\right]\left(1+k e^{i \phi}\right)}{\left(B_{\kappa}^{c} f(z)\right)^{\prime}+\lambda z\left(B_{\kappa}^{c} f(z)\right)^{\prime \prime}}-\frac{2 k e^{i \phi}\left[\left(B_{\kappa}^{c} f(z)\right)^{\prime}+\lambda z\left(B_{\kappa}^{c} f(z)\right)^{\prime \prime}\right]}{\left(B_{\kappa}^{c} f(z)\right)^{\prime}+\lambda z\left(B_{\kappa}^{c} f(z)\right)^{\prime}}\right\} \geq \eta$

Let

$F(z)=\left[\left(B_{\kappa}^{c} f(z)\right)^{\prime}+(1+2 \lambda) z\left(B_{\kappa}^{c} f(z)\right)^{\prime \prime}+\lambda z^{2}\left(B_{\kappa}^{c} f(z)\right)^{\prime \prime \prime}\right]\left(1+k e^{i \phi}\right)-2 k e^{i \phi}\left[\left(B_{\kappa}^{c} f(z)\right)^{\prime}+\lambda z\left(B_{\kappa}^{c} f(z)\right)^{\prime \prime}\right]$

and $E(z)=\left(B_{\kappa}^{c} f(z)\right)^{\prime}+\lambda z\left(B_{\kappa}^{c} f(z)\right)^{\prime \prime}$ By Lemma 1.2, equation (2.2) is equivalent to $|F(z)+(1-\eta) E(z)| \leq|F(z)-(1+\eta) E(z)|$, for $0 \leq \eta<1$.But

$$
\begin{gathered}
|F(z)+(1-\eta) E(z)|=\left|\begin{array}{l}
1-\sum_{n=2}^{\infty} n^{2}(1-\lambda+n \lambda) D(c, \kappa, n) a_{n} b_{n} z^{n-1} \\
-k i \phi\left(1-\sum_{n=2}^{\infty} n(2-n)(1-\lambda+n \lambda) D(c, \kappa, n) a_{n} b_{n} z^{n-1}\right) \\
+(1-\eta)\left(1-\sum_{n=2}^{\infty} n^{2}(1-\lambda+n \lambda) D(c, \kappa, n) a_{n} b_{n} z^{n-1}\right)
\end{array}\right| \\
\geq(2-\eta)-\sum_{n=2}^{\infty} n(n+1-\eta)(1-\lambda+n \lambda) D(c, \kappa, n) a_{n} b_{n}|z|^{n-1} \\
-k\left(1-\sum_{n=2}^{\infty} n(2-n)(1-\lambda+n \lambda) D(c, \kappa, n) a_{n} b_{n}|z|^{n-1}\right)
\end{gathered}
$$


Univalent Function defined by Bessel Function

$$
\begin{gathered}
|F(z)-(1+\eta) E(z)|=\left|\begin{array}{l}
1-\sum_{n=2}^{\infty} n^{2}(1-\lambda+n \lambda) D(c, \kappa, n) a_{n} b_{n} z^{n-1} \\
-k e^{i \phi}\left(1-\sum_{n=2}^{\infty} n(2-n)(1-\lambda+n \lambda) D(c, \kappa, n) a_{n} b_{n} z^{n-1}\right) \\
-(1+\eta)\left(1-\sum_{n=2}^{\infty} n^{2}(1-\lambda+n \lambda) D(c, \kappa, n) a_{n} b_{n} z^{n-1}\right)
\end{array}\right| \\
\leq \eta+\sum_{n=2}^{\infty} n(n+1-\eta)(1-\lambda+n \lambda) D(c, \kappa, n) a_{n} b_{n}|z|^{n-1} \\
+k\left(1-\sum_{n=2}^{\infty} n(2-n)(1-\lambda+n \lambda) D(c, \kappa, n) a_{n} b_{n}|z|^{n-1}\right)
\end{gathered}
$$

Hence, $\quad|F(z)+(1-\eta) E(z)|-|F(z)-(1+\eta) E(z)|$

$$
\begin{aligned}
& \geq(2(1-\eta)) a_{n} b_{n}|z|^{n-1}-2 \sum_{n=2}^{\infty} n(n-\eta)(1-\lambda+n \lambda) D(c, \kappa, n) a_{n} b_{n}|z|^{n-1} \\
& \left.-2 k \sum_{n=2}^{\infty} n(n-2)(1-\lambda+n \lambda) D(c, \kappa, n) a_{n} b_{n}|z|^{n-1}\right] \geq 0 .
\end{aligned}
$$

or $\sum_{n=2}^{\infty} n(n-\eta)(1-\lambda+n \lambda) D(c, \kappa, n) a_{n}+k\left[1+\sum_{n=2}^{\infty} n(n-2)(1-\lambda+n \lambda) D(c, \kappa, n) a_{n} b_{n}\right] \leq 1-\eta$.

which is equivalent to $\sum_{n=2}^{\infty}[(1-\lambda+n \lambda)[n(n-\eta)+k(n(n-2))]+k] D(c, \kappa, n) a_{n} b_{n} \leq 1-\eta$

conversely, suppose that the equation (2.1) holds good, then we have to prove that

$$
\mathfrak{R}\left\{1+\frac{z F^{\prime \prime}(z)}{F^{\prime}(z)}\left(1+k e^{i \phi}\right)-2 k e^{i \phi}\right\} \geq \eta
$$

Now choosing the values of $\mathrm{z}$ on the positive real axis where $0 \leq|z|=r<1$, the above inequality reduces to

$$
\mathfrak{R}\left\{\frac{(1-\eta)-\sum_{n=2}^{\infty}\left[n^{2}\left(1+k e^{i \phi}\right)(1-\lambda+n \lambda)-n\left(\eta+2 k e^{i \phi}\right)(1-\lambda+n \lambda)\right] D(c, \kappa, n) a_{n} b_{n} r^{n-2}+k e^{i \phi}}{1-\sum_{n=2}^{\infty}(1-\lambda+n \lambda) D(c, \kappa, n) a_{n} b_{n} r^{n-2}}\right\} \geq 0
$$

Since $\mathfrak{R}\left(e^{-i \phi}\right) \geq-\left|e^{i \phi}\right|=-1$, the above inequality reduces to

$$
\mathfrak{R}\left\{\frac{(1-\eta)-\sum_{n=2}^{\infty}\left[n^{2}(1+k)(1-\lambda+n \lambda)-n(\eta+2 k)(1-\lambda+n \lambda)\right] D(c, \kappa, n) a_{n} b_{n} r^{n-2}+k}{1-\sum_{n=2}^{\infty}(1-\lambda+n \lambda) D(c, \kappa, n) a_{n} b_{n} r^{n-2}}\right\} \geq 0
$$

Letting $r \rightarrow 1^{-}$, we get the desired result.

Corollary 2.2 Let $c>1,0 \leq \lambda<1, k \geq 0,0 \leq \eta<1$ and $z \in \mathrm{U}$, if $f \in \mathrm{UB}(\lambda, \eta, k, c)$, then 


$$
a_{n} \leq \frac{1-\eta}{k+[n(n(1+k)-(\eta+2 k))(1-\lambda+n \lambda)] D(c, \kappa, n) b_{n}} .
$$

Theorem 2.3 Let $n \geq 2, n \in \mathrm{N}, 0 \leq \eta<1, c>1,0 \leq \lambda<1, k \geq 0$ and $z \in \mathrm{U}$.If $f_{1}(z)=z$ and

$$
f_{n}(z)=z-\frac{1-\eta}{k+[n(n(1+k)-(\eta+2 k))(1-\lambda+n \lambda)] D(c, \kappa, n) b_{n}} z^{n-1},
$$

Then $f \in \mathrm{U}(\lambda, \eta, k, a, \delta)$ if and only if it can be expressed in the form

$$
f(z)=\sum_{n=2}^{\infty} \sigma_{n} f_{n}(z) \sigma_{n} \geq 0 \text { and } \sum_{n=1}^{\infty} \sigma_{n}=1 \text { or } 1=\sigma_{1}+\sum_{n=2}^{\infty} \sigma_{n}
$$

Proof. Let $f(z)=\sum_{n=2}^{\infty} \sigma_{n} f_{n}(z)$, where $\sigma_{n} \geq 0$ and $\sigma_{n}=1$ or $1=\sigma_{1}+\sum_{n=2}^{\infty} \sigma_{n}$. Then

$$
f(z)=z-\frac{1-\eta}{k+[n(n(1+k)-(\eta+2 k))(1-\lambda+n \lambda)] D(c, \kappa, n)} \sigma_{n} z^{n-1}
$$

But

$$
\begin{aligned}
f(z) & =\sum_{n=1}^{\infty}\left\{\frac{k+[n(n(1+k)-(\eta+2 k))(1-\lambda+n \lambda)] D(c, \kappa, n) b_{n}}{1-\eta}\right\} \\
& \left\{\frac{1-\eta}{k+[n(n(1+k)-(\eta+2 k))(1-\lambda+n \lambda)] D(c, \kappa, n) b_{n}} \sigma_{n}\right\} \\
& =\sum_{n=1}^{\infty} \sigma_{n}=1-\sigma_{1} \leq 1 .
\end{aligned}
$$

Using Theorem 2.1, we have $f \in \mathrm{UB}(\lambda, \eta, k, c)$.

Conversely, Let us assume that $f(z)$ is of the form (1.6) belongs to $\operatorname{UB}(\lambda, \eta, k, c$,$) . Then$

Setting

$$
a_{n} \leq \frac{1-\eta}{k+[n(n(1+k)-(\eta+2 k))(1-\lambda+n \lambda)] D(c, \kappa, n) b_{n}}, n \in \mathrm{N}, n \geq 2
$$

we have

$$
\sigma_{n}=\frac{k+[n(n(1+k)-(\eta+2 k))(1-\lambda+n \lambda)] D(c, \kappa, n)}{1-\eta} a_{n} b_{n} \text { and } \sigma_{1}=1-\sum_{n=2}^{\infty} \sigma_{n}
$$

$$
f(z)=\sum_{n=1}^{\infty} \sigma_{n} f_{n}(z)=\sigma_{1} f_{1}(z)+\sum_{n=2}^{\infty} \sigma_{n} f_{n}(z)
$$

Hence the proof.

3 Growth and Distortion Theorem

Theorem 3.1 If $f \in \mathrm{UB}(\lambda, \eta, k, c)$ and $|z|=r<1$ then

$$
r-\frac{1-\eta}{k+2(2-\eta)(1+\lambda)\left(\frac{-c}{4 \kappa}\right)} r^{2} \leq|f(z)| \leq r+\frac{1-\eta}{k+2(2-\eta)(1+\lambda)\left(\frac{-c}{4 \kappa}\right)} r^{2} .
$$

Equality in (3.1) holds true for the function $f(z)$ given by 


$$
f(z)=z-\frac{1-\eta}{k+2(2-\eta)(1+\lambda)\left(\frac{-c}{4 \kappa}\right)} z^{2} .
$$

Proof. we only prove the second part of the inequality in (3.1), since the first part can be derived by using similar arguments. If $f \in \mathrm{UB}(\lambda, \eta, k, c)$, by using Theorem 2.1 , we find that

$$
\begin{aligned}
k+2(2-\eta)(1+\lambda)\left(\frac{-c}{4 \kappa}\right) \sum_{n=2}^{\infty} a_{n} & =\sum_{n=2}^{\infty} k+2(2-\eta)(1+\lambda)\left(\frac{-c}{4 \kappa}\right) a_{n} \\
& \leq k+\sum_{n=2}^{\infty} n(n(1+k)-(\eta+2 k))(1-\lambda+n \lambda) D(c, \kappa, n) a_{n} \leq 1-\eta
\end{aligned}
$$

which readily yields the following inequality

Moreover it follows that

$$
\sum_{n=2}^{\infty} a_{n} \leq \frac{1-\eta}{k+2(2-\eta)(1+\lambda)\left(\frac{-c}{4 \kappa}\right)}
$$

$$
|f(z)|=\left|z-\sum_{n=2}^{\infty} D(c, \kappa, n) z^{n}\right| \leq|z|+|z|^{2} \sum_{n=2}^{\infty} a_{n} \leq r+r^{2} \sum_{n=2}^{\infty} a_{n} \leq r+\frac{1-\eta}{k+2(2-\eta)(1+\lambda)\left(\frac{-c}{4 \kappa}\right)} r^{2}
$$

which proves the second part of the inequality in (3.1).

Theorem 3.2 If $f \in \mathrm{UB}(\lambda, \eta, k, c$,$) and |z|=r<1$ then

$$
1-\frac{2(1-\eta)}{k+2(2-\eta)(1+\lambda)\left(\frac{-c}{4 \kappa}\right)} r \leq\left|f^{\prime}(z)\right| \leq 1+\frac{2(1-\eta)}{k+2(2-\eta)(1+\lambda)\left(\frac{-c}{4 \kappa}\right)} r
$$

Equality in (3.1) holds true for the function $f(z)$ given by (3.2)

Proof. Our proof of Theorem 3.2 is much akin to that of Theorem 3.1. Indeed, since $f \in \mathrm{UB}(\lambda, \eta, k, c)$, it is easily verified from (1.7) that

and

$$
\left|f^{\prime}(z)\right| \leq 1+\sum_{n=2}^{\infty} n a_{n}|z|^{n-1} \leq 1+r \sum_{n=2}^{\infty} n a_{n} .
$$

$$
\left|f^{\prime}(z)\right| \geq 1-\sum_{n=2}^{\infty} n a_{n}|z|^{n-1} \geq 1-r \sum_{n=2}^{\infty} n a_{n} .
$$

The assertion (3.4) of Theorem 3.2 would now follow from (3.5) and (3.6) by means of a rather simple consequence of (3.3) given by

$$
\sum_{n=2}^{\infty} n a_{n} \leq \frac{2(1-\eta)}{k+2(2-\eta)(1+\lambda)\left(\frac{-c}{4 \kappa}\right)}
$$

The completes the proof of Theorem 3.2.

4 Hadamard Product

Theorem 4.1 Let

$$
f(z)=z-\sum_{n=2}^{\infty} a_{n} z^{n} \text { and } g(z)=z-\sum_{n=2}^{\infty} b_{n} z^{n}
$$

belongs to $\mathrm{UB}(\lambda, \eta, k, c)$. Then the Hadamard Product of $f(z)$ and $g(z)$ given by 


$$
(f * g)(z)=z-\sum_{n=2}^{\infty} a_{n} b_{n} z^{n} \text { belongs to } \mathrm{UB}(\lambda, \eta, k, c) .
$$

Proof. Since $f(z)$ and $g(z)$ belongs to $\mathrm{UB}(\lambda, \eta, k, c)$, we have

$$
\sum_{n=2}^{\infty}\left\{\frac{k+[n(n(1+k)-(\eta+2 k))(1-\lambda+n \lambda)] D(c, \kappa, n) b_{n}}{1-\eta}\right\} a_{n} \leq 1 .
$$

and

$$
\sum_{n=2}^{\infty}\left\{\frac{k+[n(n(1+k)-(\eta+2 k))(1-\lambda+n \lambda)] D(c, \kappa, n) a_{n}}{1-\eta}\right\} b_{n} \leq 1 .
$$

and by applying the Cauchy-Schwarz inequality, we have

$$
\begin{gathered}
\sum_{n=2}^{\infty}\left\{\frac{\left.k+[n(n(1+k)-(\eta+2 k))(1-\lambda+n \lambda)] D(c, \kappa, n) \sqrt{a_{n} b_{n}}\right\} \sqrt{a_{n} b_{n}}}{1-\eta}\right\} \\
\leq\left(\sum_{n=2}^{\infty}\left\{\frac{(n(1+k)-(\eta+k))(1-\lambda+n \lambda) D(c, \kappa, n) b_{n}}{1-\eta}\right\} a_{n}\right)^{\frac{1}{2}} \\
\left(\sum_{n=2}^{\infty}\left\{\frac{(n(1+k)-(\eta+k))(1-\lambda+n \lambda) D(c, \kappa, n) a_{n}}{1-\eta}\right\} b_{n}\right)^{\frac{1}{2}} .
\end{gathered}
$$

However,we obtain

$$
\sum_{n=2}^{\infty}\left\{\frac{(n(1+k)-(\eta+k))(1-\lambda+n \lambda) D(c, \kappa, n) \sqrt{a_{n} b_{n}}}{1-\eta}\right\} \sqrt{a_{n} b_{n}} \leq 1 .
$$

Now we have to prove that $\sum_{n=2}^{\infty}\left\{\frac{(n(1+k)-(\eta+k))(1-\lambda+n \lambda) D(c, \kappa, n)}{1-\eta}\right\} a_{n} b_{n} \leq 1$.

$$
\begin{aligned}
& \sum_{n=2}^{\infty}\left\{\frac{(n(1+k)-(\eta+k))(1-\lambda+n \lambda) D(c, \kappa, n)}{1-\eta}\right\} a_{n} b_{n} \\
& =\sum_{n=2}^{\infty}\left\{\frac{(n(1+k)-(\eta+k))(1-\lambda+n \lambda) D(c, \kappa, n) \sqrt{a_{n} b_{n}}}{1-\eta}\right\} \sqrt{a_{n} b_{n} .}
\end{aligned}
$$

Hence the proof.

\section{Application of the Fractional Calculus}

Various operators of fractional calculus (i.e fractional derivative and fractional integral) have been rather extensively studied by many researchers(see for example [14]). Each of these theorems would involve certain operator of fractional calculus which are defined as follows.

Definition 5.1 The fractional integral operator of order $\delta$ is defined for a function $f(z)$ by

$$
D_{z}^{\delta}(f(z))=\frac{1}{\Gamma(\mu)} \int_{0}^{z} \frac{f(t)}{(z-t)^{1-\mu}} d t, \mu>0
$$

where $f(z)$ is analytic function in a simply connected region of z-plane containing the origin and the multiplicity of $(z-t)^{\mu-1}$ is removed by requiring $\log (z-t)$ to be read when $(z-t)>0$. 
Definition 5.2 The fractional derivative of order $\mu$ is defined for a function $f(z)$ by

$$
D_{z}^{\mu}(f(z))=\frac{1}{\Gamma(1-\mu)} \frac{d}{d z} \int_{0}^{z} \frac{f(t)}{(z-t)^{\mu}} d t, 0 \leq \mu<1 .
$$

where $f(z)$ is analytic function in a simply connected region of z-plane containing the origin and the multiplicity of $(z-t)^{\mu-1}$ is removed by requiring $\log (z-t)$ to be read when $(z-t)>0$.

Definition 5.3 The fractional derivative of order $k+\mu$ is defined by

$$
D_{z}^{k+\mu}(f(z))=\frac{d^{k}}{d z^{k}} D_{z}^{\mu}(f(z)), 0 \leq \mu<1 .
$$

From definition (5.1) and (5.2), after a simple computation we obtain

$$
\begin{gathered}
D_{z}^{-\mu} f(z)=\frac{1}{\Gamma(2+\mu)} z^{\mu+1}-\sum_{n=2}^{\infty} \frac{\Gamma(n+1)}{\Gamma(n+1-\mu)} a_{n} z^{n+\mu} . \\
D_{z}^{\mu} f(z)=\frac{1}{\Gamma(2-\mu)} z^{1-\mu}-\sum_{n=2}^{\infty} \frac{\Gamma(n+1)}{\Gamma(n+1-\mu)} a_{n} z^{n-\mu} .
\end{gathered}
$$

Now using equations (5.4) and (5.5), Let us prove the following theorems:

Theorem 5.4 Let $f \in \mathrm{UB}(\lambda, \eta, k, c)$. Then

$$
\begin{gathered}
\left|D_{z}^{-\mu} f(z)\right| \leq \frac{1}{\Gamma(2+\mu)}|z|^{\mu+1}\left[1+\frac{2(1-\eta)}{(2+\mu)\left[k+2(2-\eta)(1+\lambda)\left(\frac{-c}{4 \kappa}\right) b_{2}\right]}|z|^{n-1}\right] . \\
\left|D_{z}^{-\mu} f(z)\right| \geq \frac{1}{\Gamma(2+\mu)}|z|^{\mu+1}\left[1-\frac{2(1-\eta)}{(2+\mu)\left[k+2(2-\eta)(1+\lambda)\left(\frac{-c}{4 \kappa}\right) b_{2}\right]}|z|^{n-1}\right] .
\end{gathered}
$$

The inequalities (5.6) and (5.7) are attained for the function $\mathrm{f}$ given by

$$
f(z)=z-\frac{1-\eta}{k+2(2-\eta)(1+\lambda)\left(\frac{-c}{4 \kappa}\right) b_{2}} .
$$

Proof. From Theorem 2.1, we obtain

$$
\sum_{n=2}^{\infty} a_{n} \leq \frac{1-\eta}{k+2(2-\eta)(1+\lambda)\left(\frac{-c}{4 \kappa}\right) b_{2}}
$$

Using equation (5.5), we obtain

$$
\Gamma(2-\mu) z^{\mu} D_{z}^{\mu} f(z)=z-\sum_{n=2}^{\infty} \psi(n, \mu) a_{n} z^{n}
$$

such that

$$
\psi(n, \mu)=\frac{\Gamma(n+1) \Gamma(2+\mu)}{\Gamma(n+1+\mu)}, n \geq 2 .
$$

where $\psi(n, \mu)$ is a decreasing function of $\mathrm{n}$ and $0<\psi(n, \mu) \leq(2, \mu)=\frac{2}{2+\mu}$.

Using equation (5.9) and (5.10), we obtain

$$
\left|\Gamma(2-\mu) z^{\mu} D_{z}^{\mu} f(z)\right| \leq|z|+\psi(2, \mu)|z| \sum_{n=2}^{\infty} a_{n} \leq|z|+\frac{2(1-\eta)}{(2+\mu)\left[k+2(2-\eta)(1+\lambda)\left(\frac{-c}{4 \kappa}\right) b_{2}\right]}|z|^{n-1} .
$$

Which is the equation (5.6). Similarly we can get equation(5.7). 
Theorem 5.5 Let $f \in \mathrm{UB}(\lambda, \eta, k, c)$. Then

$$
\begin{gathered}
\left|D_{z}^{\mu} f(z)\right| \leq \frac{1}{\Gamma(2-\mu)}|z|^{1-\mu}\left[1+\frac{2(1-\eta)}{(2-\mu)\left[k+2(2-\eta)(1+\lambda)\left(\frac{-c}{4 \kappa}\right)\right] b_{2}}|z|^{n-1}\right] . \\
\left|D_{z}^{\mu} f(z)\right| \geq \frac{1}{\Gamma(2-\mu)}|z|^{1-\mu}\left[1-\frac{2(1-\eta)}{(2+\mu)\left[k+2(2-\eta)(1+\lambda)\left(\frac{-c}{4 \kappa}\right)\right] b_{2}}|z|^{n-1}\right] .
\end{gathered}
$$

The inequalities (5.11) and (5.12) are attained for the function $\mathrm{f}$ given by

Proof. Using equation (5.5), we obtain

$$
f(z)=z-\frac{1-\eta}{k+2(2-\eta)(1+\lambda)\left(\frac{-c}{4 \kappa}\right) b_{2}} .
$$

$$
\Gamma(2-\mu) z^{\mu} D_{z}^{\mu} f(z)=z-\sum_{n=2}^{\infty} \zeta(n, \mu) a_{n} z^{n}
$$

such that

$$
l(n, \mu)=\frac{\Gamma(n+1) \Gamma(2-\mu)}{\Gamma(n+1-\mu)}, n \geq 2 .
$$

where $\zeta(n, \mu)$ is a decreasing function of $\mathrm{n}$ and $0<\zeta(n, \mu) \leq \zeta(2, \mu)=\frac{2}{2-\mu}$.

Using equation (5.9) and (5.114), we obtain

$$
\left|\Gamma(2-\mu) z^{\mu} D_{z}^{\mu} f(z)\right| \leq|z|+\zeta(2, \mu)|z| \sum_{n=2}^{\infty} a_{n} \leq|z|+\frac{2(1-\eta)}{(2+\mu)\left[k+2(2-\eta)(1+\lambda)\left(\frac{-c}{4 \kappa}\right)\right]}|z|^{n-1} .
$$

Which is the equation (5.11). Similarly we can get equation (5.12).

Corollary 5.6 For every $f \in \mathrm{UB}(\lambda, \eta, k, c)$, we have

$$
\frac{|z|^{2}}{2}\left[1-\frac{2(1-\eta)}{3\left(k+2(2-\eta)(1+\lambda)\left(\frac{-c}{4 \kappa}\right) b_{2}\right)}|z|^{n-1}\right] \leq\left|\int_{0}^{z} f(t) d t\right|
$$

and

$$
\begin{gathered}
\leq \frac{|z|^{2}}{2}\left[1+\frac{2(1-\eta)}{3\left(k+2(2-\eta)(1+\lambda)\left(\frac{-c}{4 \kappa}\right) b_{2}\right)}|z|^{n-1}\right] \\
|z|\left[1-\frac{(1-\eta)}{k+2(2-\eta)(1+\lambda)\left(\frac{-c}{4 \kappa}\right) b_{2}}|z|^{n-1}\right] \leq|f(z)| \leq|z|\left[1+\frac{(1-\eta)}{k+2(2-\eta)(1+\lambda)\left(\frac{-c}{4 \kappa} b_{2}\right)}|z|^{n-1}\right] .
\end{gathered}
$$


Proof. By Definition 5.1 and Theorem 5.4 for $\mu=1$, we have $D_{z}^{-1} f(z)=\int_{0}^{z} f(t) d t$, the result is true.

Also by Definition 5.2 and Theorem 5.5 for $\mu=0$, we have $D_{z}^{0} f(z)=\frac{d}{d z} \int_{0}^{z} f(t) d t=f(z)$

Hence the result is true.

6 Radii of close-to-convexity Starlikeness and Convexity Theorem

Theorem 6.1 Let the function $f(z)$ defined by $f(z)=z-\sum_{n=2}^{\infty} a_{n} z^{n}$ be in the class $\operatorname{UB}(\lambda, \eta, k, c$,$) . Then$ $f(z)$ is close to convex of order $\delta(0<\delta<1)$ in $|z|<r_{1}(\lambda, \eta, k, c, \delta)$ where

$$
r_{1}(\lambda, \eta, k, c, \delta)=\inf \left\{\frac{\left[k+z-\sum_{n=2}^{\infty}[n(n(1+k)-(\eta+2 k))(1-\lambda+n \lambda)] D(c, \kappa, n) b_{n}\right](1-\delta)}{n(1-\eta)}\right\} .
$$

The result is sharp for the function $f(z)$ given by

$$
f(z)=z-\frac{1-\eta}{k+z-\sum_{n=2}^{\infty}[n(n(1+k)-(\eta+2 k))(1-\lambda+n \lambda)] D(c, \kappa, n) b_{n}} z^{n-1} .
$$

Proof. It is sufficient to show that $\left|f^{\prime}(z)-1\right| \leq 1-\delta$, for $\left.|z|<r_{1}(\lambda, \eta, k, a, \delta)\right)$.

Hence

$$
\left|f^{\prime}(z)-1\right|=\left|-\sum_{n=2}^{\infty} n a_{n} z^{n-1}\right| \leq \sum n a_{n}|z|^{n-1} \leq 1-\delta
$$

and if

$$
\sum_{n=2}^{\infty} \frac{n}{1-\delta} a_{n}|z|^{n-1} \leq 1
$$

Thus by Theorem 2.1, (6.1) will holds true if

$$
\frac{n}{1-\delta}|z|^{n-1} \leq\left\{\frac{k+z-\sum_{n=2}^{\infty}[n(n(1+k)-(\eta+2 k))(1-\lambda+n \lambda)] D(c, \kappa, n) b_{n}}{1-\eta}\right\} .
$$

(or) if $\quad|z| \leq\left\{\frac{k+z-\sum_{n=2}^{\infty}[n(n(1+k)-(\eta+2 k))(1-\lambda+n \lambda)] D(c, \kappa, n) b_{n}(1-\delta)}{n(1-\eta)}\right\}$.

The theorem follows easily from previous equation.

Theorem 6.2 Let the function $f(z)$ defined by $f(z)=z-\sum_{n=2}^{\infty} a_{n} z^{n}$ be in the class $\mathrm{UB}(\lambda, \eta, k, c$,$) . Then$ $f(z)$ is starlike of order $\delta(0<\delta<1)$ in $|z|<r_{2}(\lambda, \eta, k, c, \delta)$ where 


$$
r_{2}(\lambda, \eta, k, c, \delta)=\inf \left\{\frac{\left[k+z-\sum_{n=2}^{\infty}[n(n(1+k)-(\eta+2 k))(1-\lambda+n \lambda)] D(c, \kappa, n) b_{n}\right](1-\delta)}{(n-\delta)(1-\eta)}\right\} .
$$

The result is sharp for the function $f(z)$ given by

$$
f(z)=z-\frac{1-\eta}{k+z-\sum_{n=2}^{\infty}[n(n(1+k)-(\eta+2 k))(1-\lambda+n \lambda)] D(c, \kappa, n) b_{n}} z^{n-1} .
$$

Proof. If $f(z)$ is starlike it is sufficient to show that $\left|\frac{z f^{\prime}(z)}{f(z)}-1\right| \leq 1-\delta$, for $\left.|z|<r_{2}(\lambda, \eta, k, a, \delta),\right)$.

Since

$$
\left|\frac{z f^{\prime}(z)}{f(z)}-1\right|=\left|\frac{z\left(1-\sum_{n=2}^{\infty} n a_{n} z^{n-1}\right)}{z-\sum_{n=2}^{\infty} a_{n} z^{n}}-1\right| \leq \frac{\sum_{n=2}^{\infty}(n-1) a_{n}|z|^{n-1}}{1-\sum_{n=2}^{\infty} a_{n}|z|^{n-1}} \leq 1-\delta \text {. }
$$

Since

$$
\left|\frac{z f^{\prime}(z)}{f(z)}-1\right| \leq 1-\delta
$$

if

$$
\sum_{n=2}^{\infty} \frac{n-\delta}{1-\delta} a_{n}|z|^{n-1} \leq 1
$$

Thus by Theorem 2.1, (6.2) will holds true if

(or) if $\quad|z| \leq\left\{\frac{k+z-\sum_{n=2}^{\infty}[n(n(1+k)-(\eta+2 k))(1-\lambda+n \lambda)] D(c, \kappa, n) b_{n}(1-\delta)}{(n-\delta)(1-\eta)}\right\}^{\frac{1}{n-1}}$.

$$
\frac{n-\delta}{1-\delta}|z|^{n-1} \leq\left\{\frac{k+z-\sum_{n=2}^{\infty}[n(n(1+k)-(\eta+2 k))(1-\lambda+n \lambda)] D(c, \kappa, n) b_{n}}{1-\eta}\right\}
$$

The theorem follows easily from previous equation.

Theorem 6.3 Let the function $f(z)$ defined by $f(z)=z-\sum_{n=2}^{\infty} a_{n} z^{n}$ be in the class $\mathrm{UB}(\lambda, \eta, k, c$,$) . Then$ $f(z)$ is convex of order $\delta(0<\delta<1)$ in $|z|<r_{3}(\lambda, \eta, k, c, \delta)$ where

$$
r_{3}(\lambda, \eta, k, c, \delta)=\inf \left\{\frac{\left[k+z-\sum_{n=2}^{\infty}[n(n(1+k)-(\eta+2 k))(1-\lambda+n \lambda)] D(c, \kappa, n) b_{n}\right](1-\delta)}{n(n-\delta)(1-\eta)}\right\} .
$$

The result is sharp for the function $f(z)$ given by 


$$
f(z)=z-\frac{1-\eta}{k+z-\sum_{n=2}^{\infty}[n(n(1+k)-(\eta+2 k))(1-\lambda+n \lambda)] D(c, \kappa, n) b_{n}} z^{n-1} .
$$

Proof. Since $f(z)$ is convex it is enough to show that $\left|\frac{z f^{\prime \prime}(z)}{f^{\prime}(z)}\right| \leq 1-\delta$, for $|z|<r_{3}(\lambda, \eta, k, c, \delta$, ). Since

Thus

$$
\left|\frac{z f^{\prime \prime}(z)}{f^{\prime}(z)}\right| \leq \frac{\sum_{n=2}^{\infty} n(n-1) a_{n}|z|^{n-1}}{1-\sum_{n=2}^{\infty} a_{n}|z|^{n-1}} \leq 1-\delta
$$

if

$$
\left|\frac{z f^{\prime}(z)}{f(z)}-1\right| \leq 1-\delta \text {. }
$$

$$
\sum_{n=2}^{\infty} \frac{n(n-\delta)}{1-\delta} a_{n}|z|^{n-1} \leq 1
$$

Hence by Theorem 2.1, (6.3) will holds true if

(or) if

$$
\begin{aligned}
& \frac{n(n-\delta)}{1-\delta}|z|^{n-1} \leq\left\{\frac{k+z-\sum_{n=2}^{\infty}[n(n(1+k)-(\eta+2 k))(1-\lambda+n \lambda)] D(c, \kappa, n) b_{n}}{1-\eta}\right\} \\
& |z| \leq\left\{\frac{\left.k+z-\sum_{n=2}^{\infty}[n(n(1+k)-(\eta+2 k))(1-\lambda+n \lambda)] D(c, \kappa, n) b_{n}(1-\delta)\right)^{\frac{1}{n-1}}}{n(n-\delta)(1-\eta)}\right\}
\end{aligned}
$$

The theorem follows easily from previous equation.

\section{REFERENCES}

1. András S, and Baricz A, Monotonicity property of generalized and normalized Bessel functions of complex order, Complex Var. Elliptic Equ. 2009;54(7): 689-696.

2. Aqlan E S, Some Problems Connected with Geometric Function Theory, Ph.D. Thesis); 2004 Pune University, Pune(Unpublished).

3. Baricz A, Geometric properties of generalized Bessel functions, Publ. Math. Debrecen 2008; 73(1-2): 155178.

4. Baricz A and Ponnusamy S, Starlikeness and convexity of generalized Bessel functions, Integral Transforms Spec. Funct. 2010; 21(9-10): 641-653.

5. Baricz A, Generalized Bessel functions of the first kind, Lecture Notes in Mathematics; 1994; Springer, Berlin, 2010.

6. Bertola V, Cafaro E, Thermal instability of viscoelastic fluids in horizontal porous layers as initial value problem, Int. J. Heat Mass Transfer 2006; 49: 4003-4012.

7. Deniz E, Orhan H and Srivastava H M, Some sufficient conditions for univalence of certain families of integral operators involving generalized Bessel functions; Taiwanese J. Math. 2011; 15(2): 883-917.

8. Deniz E, Convexity of integral operators involving generalized Bessel functions, Integral Transforms Spec. Funct. 2013; 24(3): 201-216.

9. Deniz E, Differential subordination and superordination results for an operator associated with the generalized bessel functions, Arxiv 3 Apr 2012: 1204.0698v1[Math. CV].

10. Prajapat J K, Certain geometric properties of normalized Bessel functions, Appl. Math. Lett.2011; 24(12): 2133-2139. 
11. Ribeiro Jr C P, Andrade M H C, Analysis and simulation of the drying-air heating system of a Brazilian powdered milk plant, Braz. J. Chem. Eng. (2004); 21(2): 345-355.

12. Shanmugam T N, Ramachandran $C$ and Ambross Prabhu R, Certain aspects of univalent functions with negative coefficients defined by Rafid operator, Int. J. Math. Anal. (Ruse) 2013; 7(9-12): 499-509.

13. Silverman H, Univalent functions with negative coefficients, Proc. Amer. Math. Soc. 1975; 51: $109-116$.

14. Univalent functions, fractional calculus, and their applications, Ellis Horwood Series: Mathematics and its Applications, Horwood, Chichester; 1989.

15. Srivastava H M, Shanmugam T N, Ramachandran C and Sivasubramanian S, A new subclass of $k$ uniformly convex functions with negative coefficients, J. Inequal. Pure Appl. Math. 2007; 8(2):Article 43, $14 \mathrm{pp}$. 